\title{
Evaluation of the effectiveness of kinesiotaping in reducing delayed onset muscle soreness of the biceps brachii
}

\author{
Dariusz Boguszewski ${ }^{1}$, Beata Oko ${ }^{1}$, Jakub Grzegorz Adamczyk ${ }^{1,2}$, Dariusz Białoszewski ${ }^{1}$ \\ 1 Department of Rehabilitation, Physiotherapy Division, Medical University, Warsaw, Poland; ${ }^{2}$ Department of Theory \\ of Sport, Józef Piłsudski University of Physical Education, Warsaw, Poland
}

\section{Summary}

Study aim: Kinesiotaping, or Kinesiology Taping (KT), is increasingly being used for physiotherapy treatments and for biological regeneration in athletes. The aim of this study was to evaluate the effectiveness of the application of lymphatic kinesiotaping in reducing delayed onset muscle soreness of biceps brachii.

Material and methods: The study included 34 women, aged 18-27. In the test group of patients $(\mathrm{n}=17)$ a lymphatic KT application was used. All of the women performed the arm strength trial (with IPFT) and arm muscle training (with a repeat of the trial 5 times 60-80\% max). Other study tools used were an sEMG, VAS (pain assessment) and the Borg scale (subjective assessment of the intensity of effort). The measurements (arm muscles strength, sEMG, pain intensity, exercise intensity) were repeated at 24, 48, 72 and 96 hours after performing the exercise.

Results: The weakest results for the arm strength test were recorded in the second measurement in the test group, and in the third measurement in the control group. The pain level declared in the first measurement was similar in both groups. In the third measurement (48 hours after the exercise), the level of pain in the test group was significantly lower $(p<0.05)$ than in the control group.

Discussion: The kinesiotaping method can assist in reducing delayed muscle soreness, which was confirmed by the results of the tests. However, there is no evidence about the impact of specific types of KT applications on the reduction of the DOMS symptoms.

Key words: Kinesiotaping - Elastic taping - DOMS - Biceps - Electromyography - Post-exercise recovery

\section{Introduction}

Physical activity is closely associated with changes in the muscle tissue. The consequences of physical activity include symptoms of muscle fatigue, damage to muscles fibres and inflammation. The ongoing processes in these tissues often results in muscle pain. This typically appears during or after exercise. The pain observed after 24-48 hours of exercise is usually of an eccentric character, and is defined as Delayed Onset Muscle Soreness (DOMS) [10].

The level of performance of athletes depends on a properly selected training programme, as well as proper nutrition and physical regeneration. The effectiveness of the training process is confirmed by the athletes' outcomes in sports competitions, which is inextricably linked to the proper application of post-workout recovery modalities. It is biological regeneration that helps to restore the full capacity to perform physical labour. Within its framework, a number of external stimuli can be utilised to speed up this process. Among the most commonly used forms of regeneration, beyond passive rest (including sleeping), are massage treatments, physiotherapy (e.g. saunas, electrotherapy or magnetotherapy) and kinesiotherapy. Selecting the appropriate measures should be planned and individually tailored to a specific athlete, taking into account the stage in the cycle of training, and the type of exercise that is undertaken [16, 17, 27, 29].

Currently, one of the most popular treatments used by athletes to improve the quality of their motion and to aid in injury prevention is kinesiotaping $(\mathrm{KT})$. The creator of 
KT, Kenzo Kase, developed a number of applications using taping that resulted in specific actions on the tissues. One of these is the lymphatic application, which accelerates the lymph circulation, facilitates the outflow of venous blood and improves the peripheral circulation [21]. Therefore, the aim of this study was to evaluate the effectiveness of the lymphatic application of KT in reducing post-exercise soreness of the biceps muscle in healthy women.

\section{Material and methods}

The study involved 34 women, aged 18 to 27 , who were not practicing sports. They were divided randomly into two groups. The test group (1) consisted of women who underwent a kinesiotaping application. The control group (2) comprised participants who did not receive any type of treatment. The groups did not differ significantly from each other with respect to the participants' age, body mass and height (Tab. 1).

All of the women participating in the study were interviewed beforehand. The interview reviewed each woman for any cardiovascular and respiratory diseases, and for past injuries in the upper limbs and spine that would prevent them from carrying out the test. Only healthy women, with no history of these diseases and injuries, were enrolled in the study.

The test group underwent a functional test to assess their arm strength and endurance. The research tool was a test of arm strength (the International Physical Fitness Test), which involved the individuals actively hanging on the bar. The result recorded was the length of time that the individual maintained the hanging (with her head over the bar) [6].

The muscle bioelectric activity was examined by means of a surface electromyography (sEMG) using the two-channel apparatus Neuro Trac Myo Plus 2 (with Neuro Trac ETS 4.0 software). The bioelectric activity of the long head of the biceps muscle was examined at rest (60 seconds) and in isometric tension (10 seconds). Surface electrodes were placed according to the SENIAM protocol (Surface Electromyography for the Non-Invasive Assessment of Muscles), corresponding to the Recommendations of the European Guidelines for Surface
Electromyography (seniam.org) [14]. The electrodes were placed on the line between the medial acromion and the fossa cubit, at 1/3 from the fossa cubit - with a reference electrode on the wrist. The skin was cleaned before the electrodes were applied. The sEMG test result was subjected to normalisation, which was calculated as the ratio of the bioelectric activity of the muscle in suspense to the result at rest.

To assess the severity of the pain, the visual analogue scale (VAS) was used. The subjective exercise intensity was determined using the Borg Scale [11]. The pulse rate [beats/min] was measured on the radial artery of the same upper limb. Each test was performed by the same person.

All of the individuals had their arm strength tested (Measurement 1), after which an arm muscle training session was carried out. This session consisted of a series of at least five active hangs, over a period of 60 to $80 \%$ of the first test's duration (i.e. the period of the participant's maximum capabilities). Each series ended in a rest, with the length of time for the rest equal to at least twice the time of the hang. The test of arm strength and the measurements (sEMG, pain intensity, exercise intensity, heart rate) were repeated successively after 24 hours (Measurement 2), 48 hours (Measurement 3), 72 hours (Measurement 4) and 96 hours (Measurement 5). In Group 2 (the control group), the same test procedure was used as in the test group, but no physiotherapy treatment was applied.

After the completion of the test, the women who had been selected for the test group had the lymphatic applications placed on their arm muscles, in accordance with the method of KT (Fig. 1). According to Kenzo Kase, this type of application will improve the blood and lymph circulation, which results in a faster removal of metabolic products from the muscles and accelerates their regeneration [21]. The KT tapes were applied on the arms with the glenohumeral joint in an external rotation and at about a $30-45^{\circ}$ abduction with the elbow joint extended. K-Active tapes were used. All of the women in the test group were treated with two applications on each arm. The application was attached onto a previously disinfected and defatted section of skin in the form of "a fan". The base of each tape was placed, without stretching, over the lymph nodes around the front and the rear axillary notch. The tails of the tape were then attached with about a $10 \%$ strain in

Table 1. Characteristics of groups (mean values \pm standard deviations)

\begin{tabular}{lccccc}
\hline Groups & Number of people & Age [years] & Body mass [kg] & Body height [cm] & BMI [kg/m $\left.{ }^{2}\right]$ \\
\hline $\begin{array}{l}\text { Group 1 } \\
\text { (study) }\end{array}$ & $\mathrm{n}=17$ & $20.94 \pm 2.08$ & $55.53 \pm 7.95$ & $166.29 \pm 6.99$ & $20.03 \pm 2.14$ \\
$\begin{array}{l}\text { Group 2 } \\
\text { (control) }\end{array}$ & $\mathrm{n}=17$ & $20.88 \pm 2.03$ & $57.94 \pm 4.99$ & $169.53 \pm 4$ & $20.16 \pm 1.65$ \\
\hline
\end{tabular}



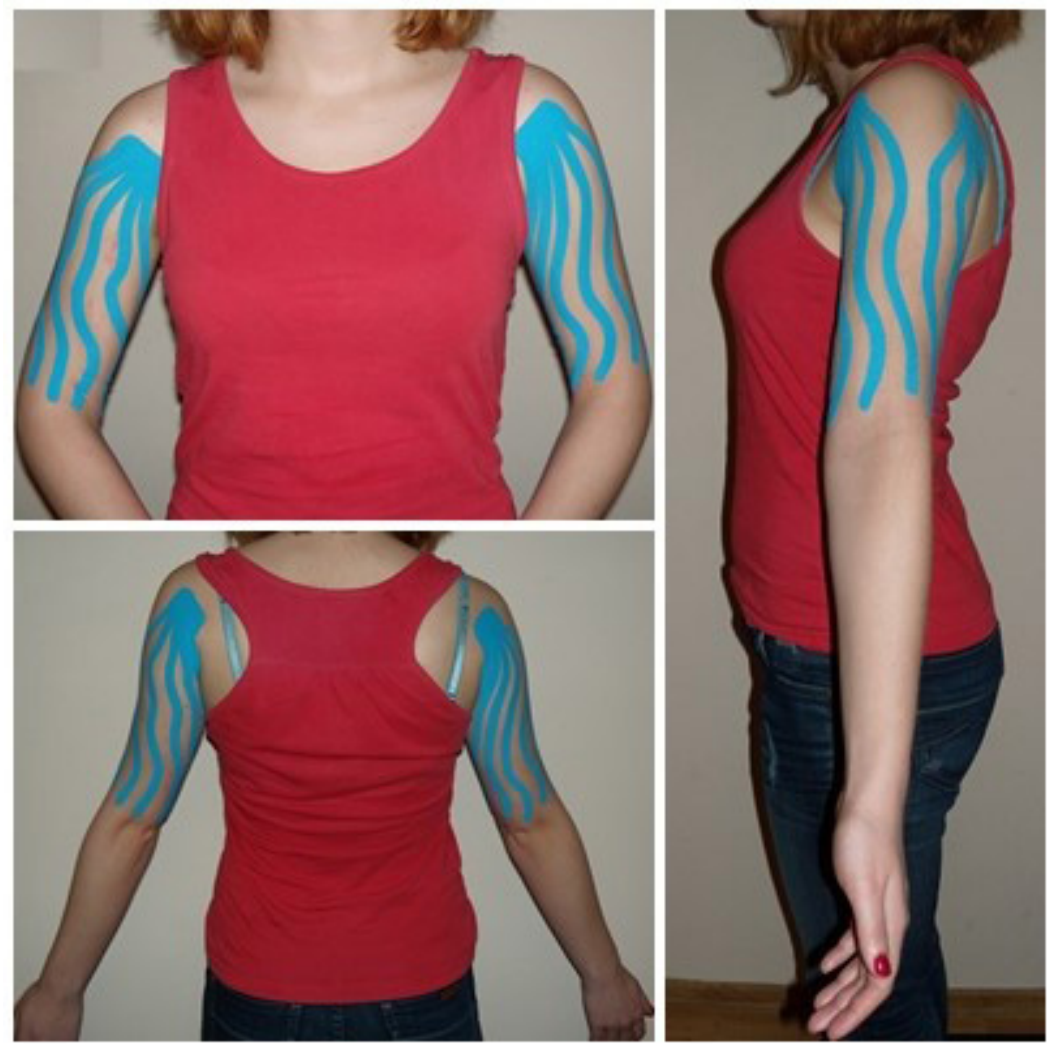

Fig. 1. Application of KT in research group

the distal direction, so that they surrounded the entire arm. The KT tapes were removed after the trial ended on the last day $[21,26]$.

Standard statistical tools of the arithmetic mean and the standard deviation were used for the analysis. The differences between individual measurements were calculated using the Wilcoxon signed-rank test; and the U MannWhitney test was used to determine the differences between the groups. The minimum level of significance was set at $\mathrm{p} \leq 0.05$.

\section{Results}

The average duration of the arm strength trial for all the women on the first day was 38.6 seconds. The lowest results in Group 1 were recorded at the second measurement; while in Group 2 they were recorded at the third measurement. In both groups, the greatest decrease in strength was recorded during the second day of the testing. In the third measurement (i.e. 48 hours after the initial

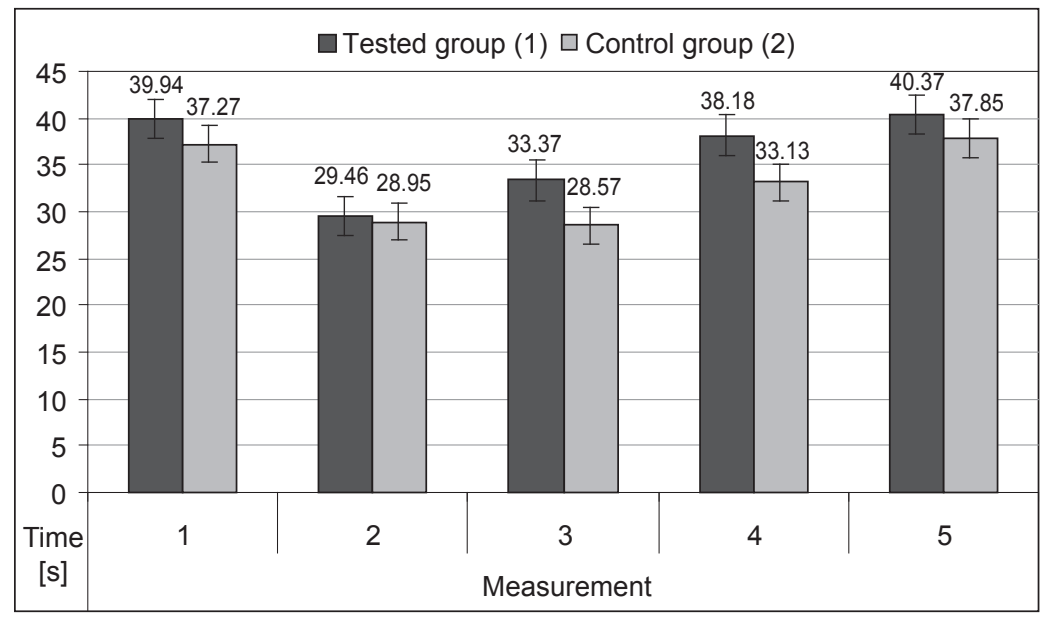

Fig. 2. Results of arm strength tests 
Table 2. Differences ( $\mathrm{p}$ ) between each measurement of arm strength test

\begin{tabular}{lccccc}
\hline \multicolumn{1}{c}{ Group (1) } & Measurement 1 & Measurement 2 & Measurement 3 & Measurement 4 & Measurement 5 \\
Group (2) & & & & & 0.479 \\
\hline Measurement 1 & 0.000 & 0.000 & 0.028 & 0.004 & 0.867 \\
Measurement 2 & 0.000 & 0.884 & & 0.019 & 0.000 \\
Measurement 3 & 0.037 & 0.109 & 0.005 & 0.002 \\
Measurement 4 & 0.730 & 0.005 & 0.000 & 0.002 & 0.380 \\
Measurement 5 & & & & \\
\hline
\end{tabular}

training session), a higher score was reported in Group 1 compared to the previous measurement; while in Group 2 the score was slightly lower (Fig. 2). On the fifth day (i.e. after 96 hours of training), the women in both groups achieved their highest test results (40.37 and $37.85 \mathrm{~s}$, respectively), although the differences as compared to the baseline measurements were not significant (Tab. 2).

Both in the test group and in the control group, a similar level of pain was reported, as measured by the VAS, during the first measurement (i.e. after three hours of a completed training session) (Fig. 3). The average result amounted to 5.65 (Group 1) and 5.53 (Group 2), respectively. In both groups, the highest pain level was recorded on the third day (Group 1: 6.88, Group 2: 7.88 - the difference was statistically significant) (Tab. 3). The lowest level of pain was observed among all women participating in the trial after 96 hours of the training session (average score in Group 1: 3.06, in Group 2: 3.59).

On the second day of testing, the highest average score (15.41) in the Borg Scale was achieved in Group 1. However, with each successive day, the women in the test group estimated the exercise intensity to be lower. Among the women in Group 2, the highest average score on the Borg Scale (16.53) was observed after the third arm strength trial. The reported estimated level of intensity decreased significantly in both groups between the third and the fourth measurements. The lowest scores were recorded on the last day of the testing (average score in Group 1: 11.94; and in

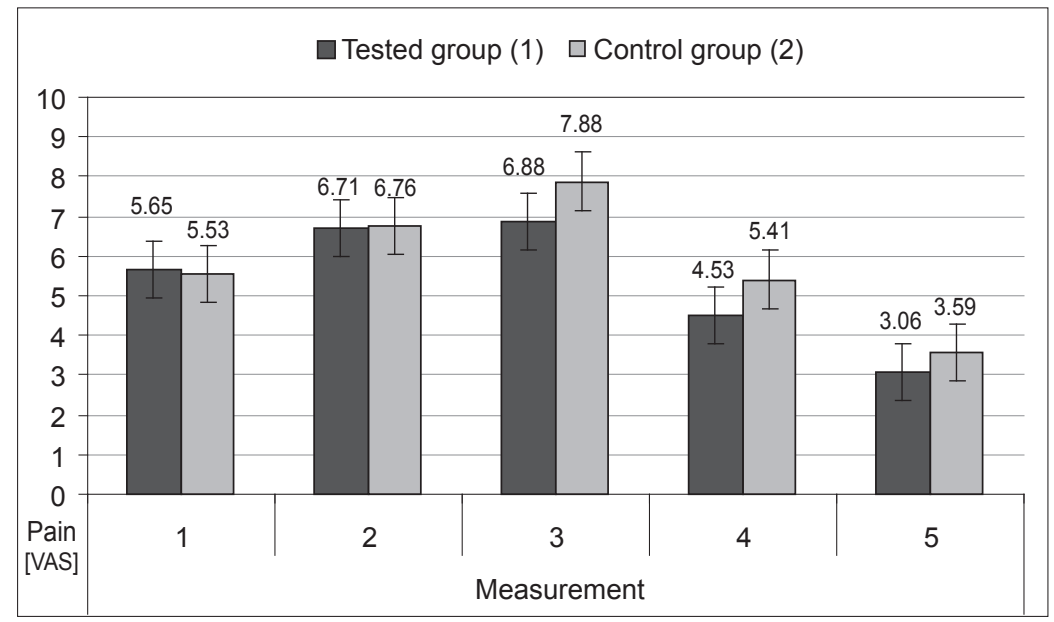

Fig. 3. Level of pain measured by VAS

Table 3. Differences (p) between each measurement of pain intensity (VAS)

\begin{tabular}{lccccc}
\hline Group 1 & Measurement 1 & Measurement 2 & Measurement 3 & Measurement 4 & Measurement 5 \\
Group 2 & & 0.037 & 0.028 & 0.023 & 0.000 \\
Measurement 1 & 0.000 & & 0.593 & 0.000 & 0.000 \\
Measurement 2 & 0.000 & 0.012 & & 0.000 & 0.000 \\
Measurement 3 & 0.791 & 0,002 & 0.000 & & 0.000 \\
Measurement 4 & 0.000 & 0.000 & 0.000 & 0.000 & \\
Measurement 5 & & & & & \\
\hline
\end{tabular}


Table 4. Normalized results of sEMG in biceps brachii (long head) before and after tests of upper limbs strength

\begin{tabular}{|c|c|c|c|c|c|c|c|c|}
\hline & & & \multicolumn{3}{|c|}{ Before } & \multicolumn{3}{|c|}{ After } \\
\hline & & & Mean value $[\%]$ & $\operatorname{Min}[\%]$ & $\operatorname{Max}[\%]$ & Mean value $[\%]$ & $\operatorname{Mmin}[\%]$ & $\operatorname{Max}[\%]$ \\
\hline \multirow{15}{*}{ 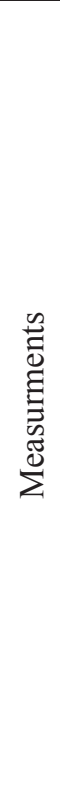 } & \multirow{3}{*}{1} & Group 1 & 0.0161 & 0.0163 & 0.0144 & 0.0155 & 0.0222 & 0.0157 \\
\hline & & Group 2 & 0.0157 & 0.0172 & 0.0178 & 0.0120 & 0.0107 & 0.0136 \\
\hline & & Difference & NS & NS & NS & NS & $\mathrm{p}<0.05$ & NS \\
\hline & \multirow{3}{*}{2} & Group 1 & 0.0174 & 0.0143 & 0.0238 & 0.0162 & 0.0122 & 0.0258 \\
\hline & & Group 2 & 0.0091 & 0.0091 & 0.0136 & 0.0090 & 0.0127 & 0.0124 \\
\hline & & Difference & $\mathrm{p}<0.05$ & NS & $\mathrm{p}<0.05$ & $\mathrm{p}<0.05$ & NS & $\mathrm{p}<0.05$ \\
\hline & \multirow{3}{*}{3} & Group 1 & 0.0168 & 0.0148 & 0.0540 & 0.0160 & 0.0144 & 0.0212 \\
\hline & & Group 2 & 0.0157 & 0.0262 & 0.0136 & 0.0089 & 0.0170 & 0.0114 \\
\hline & & Difference & $\mathrm{p}<0.05$ & $\mathrm{p}<0.05$ & $\mathrm{p}<0.05$ & $\mathrm{p}<0.05$ & NS & $\mathrm{p}<0.05$ \\
\hline & \multirow{4}{*}{4} & Group 1 & 0.0139 & 0.0114 & 0.0192 & 0.0077 & 0.0071 & 0.0096 \\
\hline & & Group 2 & 0.0073 & 0.0091 & 0.0092 & 0.0066 & 0.0073 & 0.0119 \\
\hline & & Difference & NS & NS & $\mathrm{p}<0.05$ & $\mathrm{p}<0.05$ & NS & NS \\
\hline & & Group 1 & 0.0097 & 0.0106 & 0.0110 & 0.0058 & 0.0061 & 0.0059 \\
\hline & \multirow[t]{2}{*}{5} & Group 2 & 0.0144 & 0.0231 & 0.0175 & 0.0134 & 0.0231 & 0.0147 \\
\hline & & Difference & NS & $\mathrm{p}<0.05$ & NS & NS & $\mathrm{p}<0.05$ & NS \\
\hline
\end{tabular}

Group 2: 13.41). The heart rate measurements before and after the strength trials showed no statistically significant differences between the groups.

The standardised result was obtained as a quotient of the average measurement result of the bioelectrical activity of the muscle in tension and the result at rest. The standardised result of the first sEMG test, measured before performing the exercise, was similar in both groups. However, in the second and third measurement of the sEMG, the result was significantly higher in the group of women who had the lymphatic KT applied. In both Group 1 and Group 2, a drop in the bioelectrical potential of the biceps muscle was recorded on the fourth day of the measurements. The result of the sEMG examination conducted after the arm strength trial was similar in both of the groups in the first and fifth measurements. Twenty-four hours after a completed training session, a decrease was observed in the activity of the motor units involved in the execution of the arm strength test in Group 2, which lasted up to the fifth day of testing (Tab. 4). This difference was statistically significant $(\mathrm{p}<0.05)$. After 72 hours, in all of the women, a decrease was observed in the bioelectric activity of the muscle in relation to Measurement 4.

\section{Discussion}

In the literature, one can find the results of various studies that provide evidence of the positive effects of kinesiotaping (otherwise known as dynamic taping) in sports training, injury prevention and injury treatment $[5,13,25$, 28, 30]. Wiecheć et al. [30] proved that KT applications improved the strength and muscular endurance of swimmers. In their study, the athletes demonstrated an improved jumping capability and length of distance covered, thanks to the power of inertia. This improvement in the jumping capability was also noted in a test with a group of basketball players who had recurrent inverse sprained ankles. In the group of people to whom dynamic taping was applied, a progression in the results of certain functional tests was observed. According to the authors, the KT application contributed to an improved proprioception in these athletes [28]. Also, Lumbroso et al. [24] observed an increase in the strength of the calf triceps after 15 minutes and after 48 hours of taping. Similarly, Chang et al. [13] demonstrated an increase in the subjective feeling of strength in a group of athletes using a KT application on the forearm muscles (despite a lack of reference to the results of the maximal grip strength test).

Many authors also confirm positive results with the inclusion of kinesiotaping in the process of a patient's physiotherapy $[4,9,12,15,20,22]$. For example, dynamic taping was used, among other therapies, in young football players with muscle injuries of the lower limbs. In the group of boys who included KT in their physiotherapy protocol, a faster post-traumatic pain reduction was achieved, as opposed to other players in the control group without a KT application [28].

Based on a review of the available literature, especially in the area of sports medicine, a growing interest in 
methods to support post-workout biological regeneration and to reduce delayed muscle soreness can be observed.

Kawczyński et al. [22], who examined football players before exercise and after their match, showed that there were differences in the DOMS symptoms between the dominant and non-dominant muscles of the lower limb. These results suggest that there is variability of DOMS symptoms according to the dominant side of the body, despite the use of a uniform amount of physical effort [22].

A treatment often used in minimising post-exercise muscle soreness is classical massage. Its beneficial effects in reducing pain and in restoring the strength of muscles that are burdened with an eccentric effort have been confirmed $[1,3,7,8,31]$.

The positive effect of massage and vibration training in reducing the delayed muscle soreness of flexor muscles in the elbow joint has also been proved. According to investigators, using massage helps to restore strength in the muscles (subjected to earlier exercise) more quickly, and muscle vibration causes an earlier minimisation of pain in studies where there is a comparison to a control group [19].

Valuable information with respect to methods to minimise DOMS was provided by the study results of Lee et al. [23]. In this study, the rate of the reduction of symptoms related to delayed muscle soreness among women and men was compared, while referring to the risk of ACL damage. The authors evaluated the elasticity of this ligament before and during the course of the symptoms of DOMS. Based on these results, the authors suggested that women are more vulnerable to damage in the anterior cruciate ligament, and they also return to full fitness after exercise more slowly when compared with men. Therefore, among women, the recovery time after strenuous exercise is lengthened [23].

Bae et al. [2] conducted tests on a group of 33 men to assess the impact of KT on the reduction of delayed soreness of the biceps muscle in the non-dominant upper limb. Based on the results of the thermal pain threshold, it was observed that the pain diminished significantly in the test group at 24 hours after performing the exercise. A VAS confirmed that a more rapid decrease in pain occurred in the patients with a KT application. In this case, the investigators suggested that KT causes a subjective pain reduction [2].

Surface electromyography (sEMG) is a useful and objective research tool for assessing the functional status of muscles, which has been confirmed by tests [22]. Howatson [18], using a needle EMG, attempted to answer the question of how delayed biceps soreness affects the examination of the electromyographic delay. His experiment confirmed the appropriateness of using an electromyographic examination for monitoring muscle damage [18].

The kinesiotaping method can assist in reducing delayed muscle soreness, which was confirmed by the results of the tests in the present study. However, there is no evidence about the impact of specific types of KT applications on the reduction of the DOMS symptoms. It seems reasonable to conclude that the konesiotaping method may be useful for the biological regeneration of athletes who, despite the emergence of DOMS, should not or do not want to interrupt their training exercise. Also, it should be noted that the tests included in this study were conducted on a relatively small number of people. Furthermore, the pain in response to the predetermined exercise was evaluated by means of subjective research tools. Therefore, there is a need to expand the study with a larger number of people and with males, as well as using different types of KT applications, in accordance with the principles of evidence-based medicine. Evidence for the impact of KT on the symptoms of DOMS can be achieved by means of more objective methods of evaluating and interpreting the sensation of pain and the muscle functions.

\section{Conclusions}

1. The kinesiotaping method may be beneficial in reducing post-exercise muscle soreness and enabling a faster return to physical fitness in healthy women.

2. Elastic therapeutic tape can reduce pain. Therefore, it can be suggested that the analgesic effect achieved by this technique should be used more often in rehabilitation.

3. Kinesiotaping can affect the increased activity of the motor units of the biceps muscle during post-exercise recovery. This method can be particularly effective for athletes during their preparation period before a competition, when maximal efforts are interspersed with short breaks for rest.

4. The obtained results provide the basis for continued studies in this field involving more diverse test groups and using other types of KT applications.

\section{References}

1. Arabaci R. (2008) Acute effects of pre-event lower limb massage on explosive and high speed motor capacities and flexibility. J. Sports. Sci. Med., 7: 549-555.

2. Bae S.H., Y.S. Lee, G.D. Kim, K.Y. Kim (2014) A quantitative evaluation of delayed onset muscular soreness according to application of Kinesio Taping. Advanced Science and Technology Letters, 47: 387-390.

3. Best T.M., R. Hunter, A. Wilcox, F. Haq (2008) Effectiveness of sports massage for recovery of skeletal muscle from strenuous exercise. Clin. J. Sport Med., 18(5): 446-460.

4. Białoszewski D., W. Woźniak, S. Żarek (2009) Clinical efficacy of Kinesiology Taping in reducing edema of the lower 
limbs in patients treated with the Ilizarov Method. Preliminary report. Ortop. Traumatol. Rehabil., 11(1): 46-54.

5. Bicici S., N. Karatas, G. Baltaci (2012) Effect of Athletic Taping and Kinesio Taping ${ }^{\circledR}$ on measurements of functional performance in basketball players with chronic inversion ankle sprains. Int. J. Sports Phys. Ther., 7(2): 154-166.

6. Bielski J. (1996) Life is movement. Guidebook for PE teachers. Promo-Lider, Warszawa.

7. Boguszewski D., P. Poświata, B. Oko, S. Osipiuk, J.G. Adamczyk, D. Białoszewski (2013) Assessment of sports massage effectiveness in reduction of delayed onset muscle soreness of biceps brachii. Polish J. Sport Med., 29(4): 215-221.

8. Boguszewski D., S. Szkoda, J.G. Adamczyk, D. Białoszewski (2014) Sports massage therapy on the reduction of delayed onset muscle soreness of the quadriceps femoris. Hum. Mov., 15(4): 234-237.

9. Boguszewski D., I. Tomaszewska, J.G. Adamczyk, D. Białoszewski (2015) Evaluation of effectiveness of kinesiotaping in supporting of rehabilitation of patients after meniscus injury. Preliminary report. Asian J. Med. Sci., 6(4): 61-66.

10. Bompa T.O., G.G. Haff (2009) Periodization. Theory and Methodology of Training. Human Kinetics, Champaign.

11. Borg G. (1998) Borg's Perceived Exertion and Pain Scales. Human Kinetics, Champaign.

12. Castro-Sanchez A., I. Lara-Palomo, G. Mataran-Penarrocha, M. Fernandez-Sanchez, N. Sanchez-Labraca, M. Arroyo-Morales (2012) Kinesio Taping reduces disability and pain slightly in non-specific low back pain: a randomized trial. J. Physiother, 58(2): 89-95.

13. Chang H.Y., K.Y. Chou, J.J. Lin, C.F. Lin, C.H. Wang (2010) Immediate effect of forearm Kinesio taping on maximal grip strength and force sense in healthy collegiate athletes. Phys. Ther. Sport, 11(4): 122-127.

14. European Guidelines for Surface Electromyography www.seniam.org (07.01.2014).

15. Grudzień M. (2009) Potential applications of the PNF method and kinesiotaping in the treatment of juvenile thoracic kyphosis, Acta Bio-Optica et Informatica Medi$c a, 15(2)$ : 106-107.

16. Halson S.L. (2008) Nutrition, sleep and recovery. Eur. J. Sport Sci., 8(2): 119-126.

17. Hilbert J.E., G.A. Sforzo, T. Swensen (2003) The effects of massage on delayed onset muscle soreness. Brit. J. Sport Med., 37: 72-75.

18. Howatson G. (2010) The impact of damaging exercise on electromechanical delay in biceps brachii. J. Electromyogr. Kines., 20: 477-481.

19. Imtiyaz S., Z. Veqar, M.Y. Shareep (2014) To compare the effect of vibration therapy and massage in prevention of delayed onset muscle soreness (DOMS). J. Clin. Diagn. Res., 8(1): 133-136.
20. Kalron A., S. Bar-Sela (2013) A systematic review of the effectiveness of Kinesio Taping ${ }^{\circledR}$ - Fact or fashion? Eur. J. Phys. Rehab. Med., 49(5): 699-709.

21. Kase K. (2002) Ilustrated Kinesio Taping, Ken'I - Kai Information, Tokyo.

22. Kawczyński A., D. Mroczek, P. Chmura, P. Madeleine, J. Chmura (2013) Influence of a professional football game on delayed onset muscle soreness development in dominant and non-dominant legs. Polish J. Sport Med., 29(1): 55-61.

23. Lee H., J.S. Petrofsky, M. Laymon, J. Yim (2013) A Greater Reduction of Anterior Cruciate Ligament Injury in Women Compared to Men As a Result Delayed Onset Muscle Soreness. The Thoku Journal of Experimental Medicine, 231: 111-115.

24. Lumbroso D., E. Ziv, E. Vered, L. Kalichman (2014) The effect of kinesio tape application on hamstring and gastrocnemius muscles in healthy young adults. J. Bodyw. Mov. Ther, 18: 130-138.

25. Merino-Marba R., D. Mayorga-Vega, E. Fernandez-Rodriguez (2013) Effect of Kinesio Tape application on calf pain and ankle range of motion in duathletes. J. Hum. Kin., 37: 129-135.

26. Mikołajewska E. (2011) Kinesiotaping. PZWL, Warszawa.

27. Miladi I., A. Temfemo, S.H. Mandengué, S. Ahmaidi (2011) Effect of recovery mode on exercise time to exhaustion, cardio - respiratory responses. J. Strength Cond. Res., 25(1): 205-210.

28. Ostiak W., A. Peretiatkowicz, I. Krystkowiak (2012) The effectiveness of kinesiotaping in treatment of the soft tissue injuries in adolescent football players. Post. Nauk Med., 6: 501-507.

29. Sawczyn S., W. Jagiełło, V.I. Fetisov, V.S. Mishchenko (2015) Dependence of work capacity recovery after strenuous training sessions upon individual predisposition of skilled wrestlers to work under different energy modes. Arch. Budo, 11: 197-207

30. Wiecheć M., J. Szczegielniak, J. Łuniewski, K. Bogacz, M. Krajczy, D. Banik, M. Wilk, A. Lipińska, Z. Śliwiński (2009) Evaluation of the changes of some motor features in swimmers after using Kinesiology Taping. Polish $J$. Sport Med., 25(4): 237-246.

31. Willems M.E.T., T. Hale, C.S. Wilkinson (2009) Effects of manual massage on muscle- specific soreness and single leg jump performance after downhill treadmill walking. Medicina Sportiva, 13(2): 61-66.

\section{Received 11.04.2016 \\ Accepted 15.07.2016}

(C) University of Physical Education, Warsaw, Poland 